\title{
Trial of Pimavanserin in Dementia-Related Psychosis
}

\author{
Pierre N. Tariot, M.D., Jeffrey L. Cummings, M.D., Sc.D., \\ Maria E. Soto-Martin, M.D., Ph.D., Clive Ballard, M.D., Deniz Erten-Lyons, M.D., \\ David L. Sultzer, M.D., Davangere P. Devanand, M.D., Daniel Weintraub, M.D., \\ Bradley McEvoy, Dr.P.H., James M. Youakim, M.D., \\ Srdjan Stankovic, M.D., M.S.P.H., and Erin P. Foff, M.D., Ph.D.
}

A BSTRACT

BACKGROUND

Patients with dementia due to neurodegenerative disease can have dementia-related psychosis. The effects of the oral $5-\mathrm{HT}_{2 \mathrm{~A}}$ inverse agonist and antagonist pimavanserin on psychosis related to various causes of dementia are not clear.

\section{METHODS}

We conducted a phase 3, double-blind, randomized, placebo-controlled discontinuation trial involving patients with psychosis related to Alzheimer's disease, Parkinson's disease dementia, dementia with Lewy bodies, frontotemporal dementia, or vascular dementia. Patients received open-label pimavanserin for 12 weeks. Those who had a reduction from baseline of at least $30 \%$ in the score on the Scale for the Assessment of Positive Symptoms-Hallucinations and Delusions (SAPS$\mathrm{H}+\mathrm{D}$, with higher scores indicating greater psychosis) and a Clinical Global Impression-Improvement (CGI-I) score of 1 (very much improved) or 2 (much improved) at weeks 8 and 12 were randomly assigned in a 1:1 ratio to continue receiving pimavanserin or to receive placebo for up to 26 weeks. The primary end point, assessed in a time-to-event analysis, was a relapse of psychosis as defined by any of the following: an increase of at least 30\% in the SAPS-H+D score and a CGI-I score of 6 (much worse) or 7 (very much worse), hospitalization for dementia-related psychosis, stopping of the trial regimen or withdrawal from the trial for lack of efficacy, or use of antipsychotic agents for dementia-related psychosis.

\section{RESULTS}

Of the 392 patients in the open-label phase, 41 were withdrawn for administrative reasons because the trial was stopped for efficacy; of the remaining 351 patients, $217(61.8 \%)$ had a sustained response, of whom 105 were assigned to receive pimavanserin and 112 to receive placebo. A relapse occurred in 12 of 95 patients (13\%) in the pimavanserin group and in 28 of $99(28 \%)$ in the placebo group (hazard ratio, 0.35 ; $95 \%$ confidence interval, 0.17 to $0.73 ; \mathrm{P}=0.005$ ). During the double-blind phase, adverse events occurred in 43 of 105 patients (41.0\%) in the pimavanserin group and in 41 of 112 (36.6\%) in the placebo group. Headache, constipation, urinary tract infection, and asymptomatic QT prolongation occurred with pimavanserin.

\section{CONCLUSIONS}

In a trial that was stopped early for efficacy, patients with dementia-related psychosis who had a response to pimavanserin had a lower risk of relapse with continuation of the drug than with discontinuation. Longer and larger trials are required to determine the effects of pimavanserin in dementia-related psychosis. (Funded by Acadia Pharmaceuticals; HARMONY ClinicalTrials.gov number, NCT03325556.)
From Banner Alzheimer's Institute and University of Arizona College of Medicine, Phoenix (P.N.T.); Chambers-Grundy Center for Transformative Neuroscience, Department of Brain Health, School of Integrated Health Sciences, University of Nevada, Las Vegas, Las Vegas (J.L.C.); Gérontopôle Alzheimer Clinical and Research Center, Centre Hospitalier Universitaire de Toulouse, Geriatric Department, Maintain Functions and Intrinsic Capacities with Aging Research Team, Center for Epidemiology and Population Health Research, INSERM, Université Paul Sabatier, Toulouse, France (M.E.S.-M.); University of Exeter School of Medicine, Exeter, United Kingdom (C.B.); the Department of Neurology, Oregon Health and Sciences University, Portland (D.E.-L.); the Department of Psychiatry and Human Behavior and the Institute for Memory Impairments and Neurological Disorders, University of California, Irvine, Irvine (D.L.S.), and Acadia Pharmaceuticals, San Diego (B.M., J.M.Y., S.S., E.P.F.) both in California; the Department of Psychiatry, Columbia University Irving Medical Center, New York (D.P.D.); and Perelman School of Medicine at the University of Pennsylvania, Philadelphia (D.W.). Address reprint requests to Publications-Reprints, Clinical Development, Acadia Pharmaceuticals, 502 Carnegie Center, Suite 300, Princeton, NJ 08540, or to Dr. Foff at erin.foff@acadia-pharm .com.

A complete list of investigators and collaborators in the HARMONY trial is provided in the Supplementary Appendix, available at NEJM.org.

N Engl J Med 2021;385:309-19. DOI: 10.1056/NEJMoa2034634 Copyright (๑) 2021 Massachusetts Medical Society. 
T HE MOST COMMON CAUSES OF DEMENtia - Alzheimer's disease, dementia with Lewy bodies, Parkinson's disease dementia, vascular dementia, and frontotemporal dementia $^{1}$ - may be associated with hallucinations and delusions. ${ }^{2,3}$ This syndrome of dementiarelated psychosis causes behavioral disturbances, increased caregiver burden, decreased quality of life, nursing home placement, and more rapid cognitive decline. ${ }^{4-7}$ Typical and atypical antipsychotic agents are used off-label to manage psychotic symptoms,${ }^{8}$ regardless of underlying dementia subtype, and are used cautiously in patients with dementia with Lewy bodies owing to the risk of worsening parkinsonism and other side effects. Antipsychotics may have modest short-term efficacy for dementia-related psychosis ${ }^{9-13}$ but may be associated with worsening cognition, extrapyramidal effects, sedation, falls, and metabolic abnormalities. Labels for these drugs include a warning for increased risk of death among elderly patients. This situation has contributed to clinical guidelines that focus on minimizing the use of antipsychotics, discontinuing treatment when improvement is not observed, and reassessing continued use after initial improvement. ${ }^{3}$

Pimavanserin is a serotonin-receptor modulator that acts primarily as a selective 5-hydroxytryptamine receptor subtype $2 \mathrm{~A}\left(5-\mathrm{HT}_{2 \mathrm{~A}}\right)$ inverse agonist and antagonist, with lesser activity at $5-\mathrm{HT}_{2 \mathrm{C}}$ and no appreciable activity at other receptors in vitro. This profile is different from those of conventional antipsychotics, which bind to D2 dopamine receptors and have varying activity at other receptors, including at histaminergic and muscarinic receptors. ${ }^{14}$

In a 6 -week trial of pimavanserin for the treatment of hallucinations and delusions associated with Parkinson's disease-related psychosis, ${ }^{15}$ a subgroup of patients with cognitive impairment had a change from baseline in the psychosis score with pimavanserin that was better than the change observed in cognitively unimpaired patients. ${ }^{16}$ In a brief, double-blind, placebo-controlled trial involving patients with possible or probable Alzheimer's disease-related psychosis, more than half of whom also had cerebrovascular disease, pimavanserin resulted in a larger effect in patients with more severe psychosis. ${ }^{17}$

On the basis of these preliminary findings in patients with psychosis due to Parkinson's disease or Alzheimer's disease, the current HARMONY trial was initiated as a phase 3, randomized discontinuation trial of the safety and efficacy of pimavanserin for the treatment of delusions and hallucinations associated with several common forms of dementia. The randomized discontinuation design has been used in a trial of risperidone involving patients with Alzheimer's disease and behavioral complications. ${ }^{18}$ This design addresses the question of whether treatment with pimavanserin would confer sustained benefits with respect to psychosis in patients with an initial response to the drug; the design also translates to decisions about medication discontinuation and minimizes exposure to ineffective therapy.

METHODS

\section{TRIAL DESIGN AND OVERSIGHT}

The trial was conducted at 101 clinical sites in North America, eastern and western Europe, and Latin America from August 2017 through October 2019. The trial included a 5-week screening period, a 12-week open-label period, a doubleblind treatment period of up to 26 weeks, and a 4-week safety follow-up period (Fig. S1 in the Supplementary Appendix, available with the full text of this article at NEJM.org). During screening, patients received brief psychosocial therapy, ${ }^{19}$ a simple psychological therapy tailored to each patient and partner to encourage frequent social interaction and engagement in activities of interest. This technique was used to follow best practices that encourage initiation of nonpharmacologic intervention for dementia and psychosis and to ensure that only patients with persistent psychosis that warranted pharmacologic treatment were enrolled.

Open-label treatment was initiated with pimavanserin at a dose of $34 \mathrm{mg}$ once daily; dose reduction to $20 \mathrm{mg}$ daily on the basis of side effects was permitted from weeks 1 to 4 , after which the dose remained fixed for the remainder of the open-label phase. Patients who met prespecified criteria for treatment response were randomly assigned to continue receiving pimavanserin at their final open-label dose or to receive placebo and were regularly evaluated for relapse of psychosis. Identical tablets and packaging were provided for pimavanserin and placebo.

A pregenerated permuted-block schedule was used to randomly assign patients in a 1:1 ratio 
to receive pimavanserin or placebo, with stratification according to dementia subtype (Alzheimer's disease or frontotemporal dementia vs. vascular dementia vs. Parkinson's disease dementia or dementia with Lewy bodies) and geographic region. The investigators and site staff, who assessed clinical responses; the members of the adjudication committee, who determined whether the criteria for protocol-defined relapse were met; and sponsor personnel were unaware of the trial-group assignments.

The trial protocol (available at NEJM.org) was approved by an independent ethics committee or institutional review board at each trial site and implemented following the principles of Good Clinical Practice derived from the Declaration of Helsinki and in accordance with local regulations and International Council for Harmonisation guidelines. All the patients or their authorized representatives provided written informed consent before any trial procedures.

The sponsor, Acadia Pharmaceuticals, designed and conducted the trial, provided the active drug and placebo, and performed the statistical analysis. All the authors vouch for the completeness and accuracy of the data, for the complete reporting of adverse events, and for the fidelity of the trial to the protocol. The first draft of the manuscript was written primarily by the last author, with assistance from a medical writer who was paid by the sponsor. There were confidentiality agreements in place between the authors and the sponsor, but there were no restrictions on the academic authors in submitting the trial results for publication.

\section{PATIENTS}

Adults 50 to 90 years of age were eligible if they met criteria for dementia (National Institute on Aging-Alzheimer's Association ${ }^{20}$ ) and met clinical criteria for one or more of the following: Parkinson's disease dementia, ${ }^{21}$ dementia with Lewy bodies, ${ }^{22}$ possible or probable Alzheimer's disease,${ }^{20}$ frontotemporal dementia, ${ }^{23}$ or vascular dementia. ${ }^{24}$ Patients were required to have a score of 6 to 24 on the Mini-Mental State Examination (MMSE; range, 0 to 30 ; with higher scores indicating better performance), ${ }^{25}$ psychotic symptoms for at least 2 months, and a care partner who was able to reliably report patient symptoms. At screening and baseline, patients were required to have a total score on the Scale for the Assessment of Positive Symptoms (SAPS)-Hal- lucinations and Delusions (SAPS-H+D) of 10 or more, ${ }^{26}$ a SAPS-H+D global rating subscore of 4 or more, and a Clinical Global ImpressionSeverity (CGI-S) ${ }^{27}$ score of 4 or more (range, 1 to 7 , with higher scores indicating greater impairment). The SAPS $-\mathrm{H}+\mathrm{D}$ is a subset of the SAPS, which was devised for the assessment of schizophrenia symptoms; the range is 0 to 100 , and the scale contains 7 hallucination items (6 individual items and 1 global item) and 13 delusion items (12 individual items and 1 global item), each with a range of 0 to 5 and with higher scores indicating more severe symptoms. ${ }^{26} \mathrm{~Pa}$ tients who were receiving an acetylcholinesterase inhibitor or memantine had to be receiving a stable dose for 12 weeks before screening, and the use of antipsychotics was prohibited for 2 weeks or five half-lives before baseline and during the trial. Full eligibility criteria are provided in the protocol.

\section{TRIAL ASSESSMENTS}

The SAPS-H+D and CGI-S scores were assessed at each clinic visit in the open-label and doubleblind phases. In the double-blind phase, clinic visits were at weeks 13,14, 16, 18, 22, 26, 30, 34, and 38. Physical and neurologic examination with vital signs, clinical laboratory testing, and 12-lead electrocardiography were performed at screening and during the open-label and doubleblind phases (Fig. S1). The MMSE and Global Clinician Assessment of Suicidality were conducted at each clinic visit, and the score on the Extrapyramidal Symptom Rating Scale-Abbreviated (ESRS-A; range, 0 to 120 , with higher scores indicating greater motor dysfunction $)^{28}$ was assessed at baseline, week 12 of the openlabel phase, and at each visit during the doubleblind phase.

Response criteria were assessed at weeks 8 and 12 of the open-label phase, and response was required at both visits in order for the patient to proceed in the trial; thus, the week 12 population was enriched with patients who had a response to pimavanserin. Response was defined as both a reduction (improvement) from baseline of at least 30\% in the SAPS-H+D total score and a CGI-Improvement (CGI-I) score ${ }^{27}$ of 1 (very much improved) or 2 (much improved) relative to baseline at both weeks 8 and 12. Patients who met these criteria entered the double-blind phase. Assessment for relapse occurred throughout the double-blind phase. Discontinuations in the 
double-blind phase were adjudicated by an independent committee to determine whether the criteria for protocol-defined relapse were met.

\section{END POINTS}

The primary end point was the time from randomization to relapse of psychosis, as defined as one or more of the following: an increase from baseline (double-blind phase) of at least 30\% in the SAPS-H+D total score and a CGI-I score of 6 (much worse) or 7 (very much worse), hospitalization due to dementia-related psychosis, stopping the trial regimen or withdrawal from the trial owing to lack of efficacy, or the use of other antipsychotics for the treatment of dementia-related psychosis. The data and safety monitoring board interrogated the data at the interim analysis to determine whether prespecified efficacy stopping criteria had been met.

The secondary end point was the time from randomization to trial discontinuation for any reason. Exploratory end points included the CGI-I score and the change from baseline in the doubleblind phase in scores on the following scales: SAPS-H+D, SAPS-Hallucinations (SAPS-H), SAPSDelusions (SAPS-D), CGI-S, Zarit Burden Interview, Karolinska Sleepiness Scale, and EuroQol Group 5-Dimension 5-Level questionnaire. (Details on these scales are provided in Section 6.2 in the protocol.)

\section{STATISTICAL ANALYSIS}

The sample-size calculation assumed 75 postrandomization relapse events on the basis of an assumed hazard ratio of 0.47 , an overall twosided alpha level of 0.05 , a power of $90 \%$, and use of the O'Brien-Fleming stopping boundary for a single interim analysis. A two-sided $\mathrm{P}$ value of 0.0066 for the hazard ratio for the betweengroup difference in time to relapse was established as the value that justified stopping the trial at the interim analysis.

The primary efficacy analysis was based on the intention-to-treat population, defined as all the patients who underwent randomization. The time from randomization to relapse in the double-blind phase was compared between trial groups with the use of a Cox regression model with covariates for trial-group assignment, dementia strata, and geographic region and with a robust sandwich variance-type estimator. ${ }^{29} \mathrm{We}$ tested the assumptions of the primary analysis with the use of a global goodness-of-fit test based on Schoenfeld residuals. There was no evidence that the assumptions were violated for the primary and key secondary end points. Data for patients who did not have a relapse event were censored at the time of their last assessment before the date of data cutoff for the interim analysis. The secondary end point, time to trial discontinuation for any reason, was tested at the same alpha level and analyzed with the use of the Cox regression model as described for the primary end point. A hierarchical testing procedure was used to control the overall type I error rate for the primary and secondary end points at a significance level of $\mathrm{P}<0.0066$ for each step. There was no plan for imputation of missing data; however, a sensitivity analysis was planned to investigate the assumption of noninformative censoring for the primary efficacy end point that imputed possible outcomes for the patients who had discontinued participation in the trial without having had a relapse, as well as additional sensitivity analyses that included events up to trial discontinuation and the end of the trial. The statistical analysis plan is available with the protocol at NEJM.org.

\section{RESULTS}

\section{OPEN-LABEL PHASE}

A total of 794 patients were screened during approximately 24 months of trial enrollment (Fig. 1), and 392 patients were enrolled in the open-label phase. The distribution of dementia diagnoses was as follows: $66.3 \%$ of the patients had Alzheimer's disease, $15.1 \%$ had Parkinson's disease dementia, 9.7\% had vascular dementia, 7.1\% had dementia with Lewy bodies, and 1.8\% had frontotemporal dementia. The mean $( \pm S D)$ age of the enrolled patients was $74.5 \pm 8.3$ years, the mean duration of cognitive impairment was $4.3 \pm 2.8$ years, $95.2 \%$ of the patients were living at home (Table 1), and $81.6 \%$ had both delusions and hallucinations at baseline. The mean MMSE score was $16.7 \pm 4.7$. The mean baseline SAPS$\mathrm{H}+\mathrm{D}$ score was $24.4 \pm 9.2$, and the mean CGI-S score for psychosis was $4.7 \pm 0.7$, consistent with moderate psychosis. A total of $46.4 \%$ of the pa- 


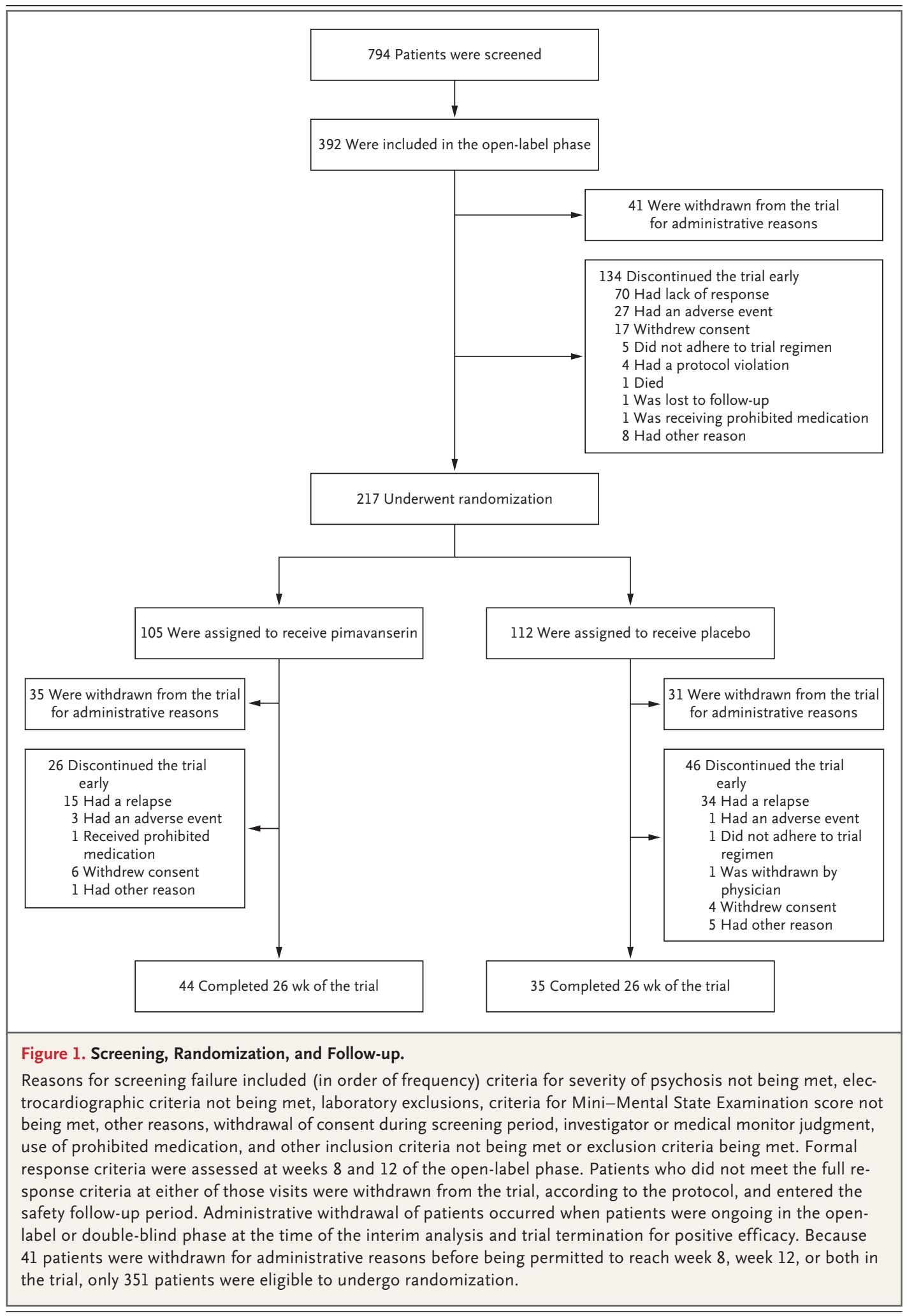

N ENGLJ MED 385;4 NEJM.ORG JULY 22, 2021

The New England Journal of Medicine

Downloaded from nejm.org at UNIVERSITY OF EXETER on October 22, 2021. For personal use only. No other uses without permission. Copyright () 2021 Massachusetts Medical Society. All rights reserved. 


\begin{tabular}{|c|c|c|c|}
\hline \multirow[t]{2}{*}{ Characteristic } & \multirow{2}{*}{$\begin{array}{l}\text { Open-Label Phase } \hat{\dagger} \\
\text { Pimavanserin } \\
(\mathrm{N}=392)\end{array}$} & \multicolumn{2}{|c|}{ Double-Blind Phase } \\
\hline & & $\begin{array}{l}\text { Pimavanserin } \\
\quad(\mathrm{N}=105)\end{array}$ & $\begin{array}{l}\text { Placebo } \\
(\mathrm{N}=112)\end{array}$ \\
\hline \multicolumn{4}{|l|}{ Age $-y r$} \\
\hline Mean & $74.5 \pm 8.3$ & $73.8 \pm 8.4$ & $74.9 \pm 8.6$ \\
\hline Range & $52-90$ & $53-89$ & $52-90$ \\
\hline Female sex — no. (\%) & $229(58.4)$ & $62(59.0)$ & $69(61.6)$ \\
\hline White race - no./total no. $(\%) \ddagger$ & $371 / 384(96.6)$ & $103 / 105(98.1)$ & $107 / 109(98.2)$ \\
\hline Hispanic or Latino ethnic group — no./total no. (\%) & $86 / 384(22.4)$ & $25 / 105(23.8)$ & $26 / 109$ (23.9) \\
\hline Living at home - no. (\%) & $373(95.2)$ & $94(89.5)$ & $109(97.3)$ \\
\hline Age at onset of cognitive impairment $-y r$ & $70.6 \pm 8.9$ & $70.1 \pm 9.1$ & $71.2 \pm 9.2$ \\
\hline Duration of cognitive impairment $-\mathrm{yr}$ & $4.3 \pm 2.8$ & $4.2 \pm 2.2$ & $4.2 \pm 3.1$ \\
\hline \multicolumn{4}{|l|}{ History of psychotic symptoms — no./total no. (\%) } \\
\hline Auditory hallucinations & $287 / 382(75.1)$ & $81 / 101(80.2)$ & $92 / 111(82.9)$ \\
\hline Visual hallucinations & $302 / 382(79.1)$ & $77 / 101(76.2)$ & $92 / 111(82.9)$ \\
\hline Delusions & $319 / 382(83.5)$ & $77 / 101(76.2)$ & $96 / 111(86.5)$ \\
\hline \multicolumn{4}{|l|}{ Primary dementia subtype - no. (\%) } \\
\hline Alzheimer's disease & $260(66.3)$ & $67(63.8)$ & $70(62.5)$ \\
\hline Dementia with Lewy bodies & $28(7.1)$ & $6(5.7)$ & $4(3.6)$ \\
\hline Frontotemporal dementia & $7(1.8)$ & $1(1.0)$ & $2(1.8)$ \\
\hline Parkinson's disease dementia & $59(15.1)$ & $19(18.1)$ & $23(20.5)$ \\
\hline Vascular dementia & $38(9.7)$ & $12(11.4)$ & $13(11.6)$ \\
\hline \multicolumn{4}{|l|}{ Geographic region — no. (\%) } \\
\hline North America & $118(30.1)$ & $28(26.7)$ & $29(25.9)$ \\
\hline Latin America & $27(6.9)$ & $7(6.7)$ & $9(8.0)$ \\
\hline Eastern Europe & $209(53.3)$ & $65(61.9)$ & $67(59.8)$ \\
\hline Western Europe & $38(9.7)$ & $5(4.8)$ & $7(6.2)$ \\
\hline \multicolumn{4}{|l|}{ Antidementia drug use - no. (\%) $\int$} \\
\hline Any & $273(69.6)$ & $81(77.1)$ & $72(64.3)$ \\
\hline Acetylcholinesterase inhibitor & $172(43.9)$ & 49 (46.7) & $42(37.5)$ \\
\hline Antidepressant use - no. (\%) & $81(20.7)$ & $14(13.3)$ & $23(20.5)$ \\
\hline MMSE total score & $16.7 \pm 4.7$ & $18.3 \pm 5.4$ & $17.9 \pm 5.9$ \\
\hline SAPS-H+D score $\|$ & $24.4 \pm 9.2$ & $5.0 \pm 5.3$ & $5.2 \pm 5.4$ \\
\hline CGI-S score $* *$ & $4.7 \pm 0.7$ & $2.3 \pm 1.0$ & $2.3 \pm 1.0$ \\
\hline
\end{tabular}

* Plus-minus values are means \pm SD. Percentages may not total 100 because of rounding.

$\dagger$ The patients who did not undergo randomization had several reasons for not doing so, including 41 patients who were withdrawn for administrative reasons at the time of trial discontinuation and who were therefore not given the opportunity to complete the open-label phase. The 175 patients who did not undergo randomization, in aggregate, had the following characteristics: female sex, $56.0 \%$; mean $( \pm S D)$ age, $74.6 \pm 8.0$; White race, $94.7 \%$; mean $( \pm S E)$ score on the Mini-Mental State Examination (MMSE) (described below), 16.5 \pm 5.1 ; and mean $( \pm \mathrm{SE}$ ) score on the Scale for the Assessment of Positive Symptoms-Hallucinations and Delusions (SAPS-H+D) (described below), 23.1 $1 \pm 8.7$.

$\mp$ Race and ethnic group were determined by site staff or by the patient or care partner.

$\int$ Antidementia drugs included acetylcholinesterase inhibitors, memantine, or both. The use of an acetylcholinesterase inhibitor alone or in a combination pill is noted as a subcategory.

9 The MMSE is a brief 30-point questionnaire that is used to quantitatively assess cognition. ${ }^{25}$ Scores range from 0 to 30 , with higher scores indicating higher performance.

$\|$ The SAPS-H+D consists of 20 items, including 7 hallucination items ( 6 individual items and 1 global item) and 13 delusion items ( 12 individual items and 1 global item). Scores on each item range from 0 to 5 (total scores range from 0 to 100, with higher scores indicating a greater degree of psychosis).

$* *$ The Clinical Global Impression-Severity (CGI-S) scale is a clinician-rated, 7-point scale that is designed to rate the severity of the patient's hallucinations and delusions at the time of assessment using the investigator's judgment and past experience with patients who have the same disorder (i.e., dementia-related psychosis). ${ }^{27}$ Scores range from 1 to 7, with higher scores indicating greater impairment. 
tients were taking at least five concomitant medications, and $46.9 \%$ had at least five coexisting medical conditions.

Pimavanserin at a dose of $20 \mathrm{mg}$ was administered to 31 of 392 patients (7.9\%) in the openlabel phase, and the 34-mg dose was administered to the other patients. A total of 41 patients remained in the open-label portion of the trial at the time of trial discontinuation and were withdrawn for administrative reasons. Of 351 remaining eligible patients, 217 (61.8\%) had a sustained response and were randomly assigned to continue receiving pimavanserin or to receive placebo. A total of 70 patients (19.9\%) did not meet the criteria for a response at week 8 , week 12 , or both and were not carried over to the randomized portion of the trial; 27 (7.7\%) discontinued owing to an adverse event; 1 (0.3\%) died; and 36 had other reasons for discontinuing (Fig. 1).

The mean SAPS $-\mathrm{H}+\mathrm{D}$ scores at weeks 2, 4, and 8 of the open-label phase are shown in Figure S2A. Among patients who were assessed at week 12 , there was a $75.2 \%$ mean reduction from baseline in the SAPS-H+D score (Table S1). Changes from baseline in the open-label phase through week 12 in the SAPS-H, SAPS-D, and CGI-S scores are also shown in Table S1. The proportion of patients who met full response criteria increased over time (Fig. S2B).

\section{DOUBLE-BLIND PHASE}

At the time of the interim analysis, 194 patients had undergone randomization (95 to pimavanserin and 99 to placebo); at the time of trial discontinuation, 23 additional patients had entered the double-blind phase (total, 105 in the pimavanserin group and 112 in the placebo group). Disease characteristics were similar in the two trial groups (Table 1). The median duration of exposure in the double-blind phase was 17.7 weeks with pimavanserin and 10.9 weeks with placebo. By the end of the trial, 72 patients (26 in the pimavanserin group and 46 in the placebo group) had discontinued the trial early during the double-blind phase; the most common reasons were relapse of psychosis (15 patients in the pimavanserin group and 34 in the placebo group) and withdrawal of consent ( 6 patients in the pimavanserin group and 4 in the placebo group). A total of 79 patients completed the 26-week dou- ble-blind phase (44 in the pimavanserin group and 35 in the placebo group), and 66 patients (35 in the pimavanserin group and 31 in the placebo group) who remained in the double-blind portion were withdrawn for administrative reasons because the trial was stopped early at the interim analysis (Fig. 1).

At the time of the interim analysis, the frequency of relapse was 13\% in the pimavanserin group and $28 \%$ in the placebo group (hazard ratio for time to relapse, $0.35 ; 95 \%$ confidence interval [CI], 0.17 to $0.73 ; \mathrm{P}=0.005$ ). The significance level for the Cox regression analysis was below the prespecified stopping boundary of 0.0066 , and the trial was stopped (Table 2). The risk of relapse over time is shown in Figure 2. Prespecified sensitivity analyses, including in the full enrolled population and per-protocol population, are shown in Table S2. Trial discontinuation for any reason occurred in 21 patients (22\%) in the pimavanserin group and in 38 patients (38\%) in the placebo group (hazard ratio for time to trial discontinuation for any reason, $0.45 ; 95 \% \mathrm{CI}, 0.26$ to $0.79 ; \mathrm{P}=0.005$ ) (Table 2). There were no missing data for the primary or secondary end points.

To assess the effect on specific measures known to be affected by antipsychotics in this trial, patients were regularly assessed for cognitive function (with the use of the MMSE) and motor function (with the use of the ESRS-A). During the open-label phase, the mean $( \pm S E)$ change in the total MMSE score was $1.0 \pm 0.2$ points, and the mean change from baseline in the ESRS-A score was $-0.7 \pm 0.2$ points, with both changes indicating improvement (Figs. S4 and S5). During the double-blind phase, the mean change in the MMSE score did not differ substantially between patients who received pimavanserin and those who received placebo, and the mean change from baseline was $1.2 \pm 0.5$ in patients treated with pimavanserin for the 38week duration of the trial (Fig. S4). During the double-blind phase, the mean change in the ESRS-A score at 26 weeks was $-0.9 \pm 0.6$ with pimavanserin and $-0.4 \pm 0.3$ with placebo, favoring pimavanserin (Fig. S5).

\section{ADVERSE EVENTS AND SAFETY}

During the open-label phase, 142 patients (36.2\%) had a treatment-emergent adverse event, defined 


\begin{tabular}{|c|c|c|c|c|}
\hline End Point & Pimavanserin $(\mathrm{N}=95)$ & Placebo $(\mathrm{N}=99)$ & Hazard Ratio $(95 \% \mathrm{CI})$ & P Value \\
\hline \multicolumn{5}{|l|}{ Primary end point: relapse of psychosis } \\
\hline Patients who had a relapse event — no. (\%) & $12(13)$ & $28(28)$ & $0.35(0.17-0.73)$ & 0.005 \\
\hline Patients with censored data - no. (\%) & $83(87)$ & $71(72)$ & & \\
\hline Completed wk 26 without a relapse & $37(39)$ & $28(28)$ & & \\
\hline Prematurely discontinued before wk 26 & $9(9)$ & $10(10)$ & & \\
\hline Were continuing in trial at time of data cutoff & $37(39)$ & $33(33)$ & & \\
\hline \multicolumn{5}{|l|}{ Secondary end point: trial discontinuation } \\
\hline Patients who discontinued for any reason - no. (\%) & $21(22)$ & $38(38)$ & $0.45(0.26-0.79)$ & 0.005 \\
\hline Patients with censored data - no. (\%) & $74(78)$ & $61(62)$ & & \\
\hline Completed wk 26 without a relapse & $37(39)$ & $28(28)$ & & \\
\hline Were continuing in trial at time of data cutoff & $37(39)$ & $33(33)$ & & \\
\hline
\end{tabular}

* Shown are the results at the interim analysis among the 95 patients who were randomly assigned to continue receiving pimavanserin and the 99 patients who were assigned to receive placebo during the double-blind phase. The primary and secondary end points, which were assessed in time-to-event analyses, were tested hierarchically at the two-sided 0.0066 level to control trial-wise type I error, with relapse of psychosis being tested first.

as an adverse event with an onset on or after the date of the first trial dose and no later than the date of the last trial dose plus 30 days. Such events that occurred in more than $2 \%$ of the patients in the open-label phase were urinary tract infection (in $5.1 \%$ of patients), constipation (in 2.6\%), and hypertension (in 2.3\%) (Table 3). Serious adverse events occurred in 20 patients (5.1\%) in the open-label phase (Table S3). One patient $(0.3 \%)$ died from suspected myocardial infarction that was considered by the investigator to be unrelated to pimavanserin. In addition to the investigator's opinion, the case was internally reviewed by the sponsor's pharmacovigilance group and was also reviewed by the independent data and safety monitoring board; neither group proposed modification to the investigator's determination of causality. No clinically significant mean changes in clinical laboratory values or vital signs were observed. The mean $( \pm \mathrm{SE})$ prolongation of the corrected QT interval, calculated with the use of Fridericia's formula $(\mathrm{QTcF})$, during the open-label phase was $5.4 \pm 0.9 \mathrm{msec} ; 1$ patient $(0.3 \%)$ had an asymptomatic increase in the QTcF of more than $60 \mathrm{msec}$ with pimavanserin. In total, five adverse events involving an asymptomatic prolongation of the QT interval were reported with pimavanserin (two in the open-label phase and three in the doubleblind phase), affecting $1.3 \%$ of the patients.

Common adverse events (occurring in $\geq 3 \%$ of the patients in either group) were headache (in
$9.5 \%$ of patients in the pimavanserin group and in $4.5 \%$ in the placebo group), urinary tract infection (in $6.7 \%$ and $3.6 \%$, respectively), and nasopharyngitis (in 1.0\% and 3.6\%) (Table 3). Among all the patients receiving pimavanserin during the trial, the most common adverse events were constipation (in 3.1\%), headache (in 4.1\%), and urinary tract infection (in 6.4\%). In the double-blind phase, the incidence of serious adverse events (Table S4) and adverse events leading to trial discontinuation (Table 3) was similar in the two trial groups. One patient in the pimavanserin group died during the doubleblind phase from septic and metabolic encephalopathy caused by a dental abscess. No clinically significant differences were observed between the pimavanserin group and the placebo group in mean laboratory values or vital signs during this phase of the trial. Two patients (one in each group) had asymptomatic QTcF values greater than 500 msec.

\section{DISCUSSION}

This randomized discontinuation trial examined sustained response to pimavanserin treatment followed by the effect of treatment discontinuation on recurrence of psychosis in patients with several types of neurodegenerative disease. After randomization, the percentage of patients who had a relapse of psychosis was 13\% among those who continued to receive pimavanserin and $28 \%$ 


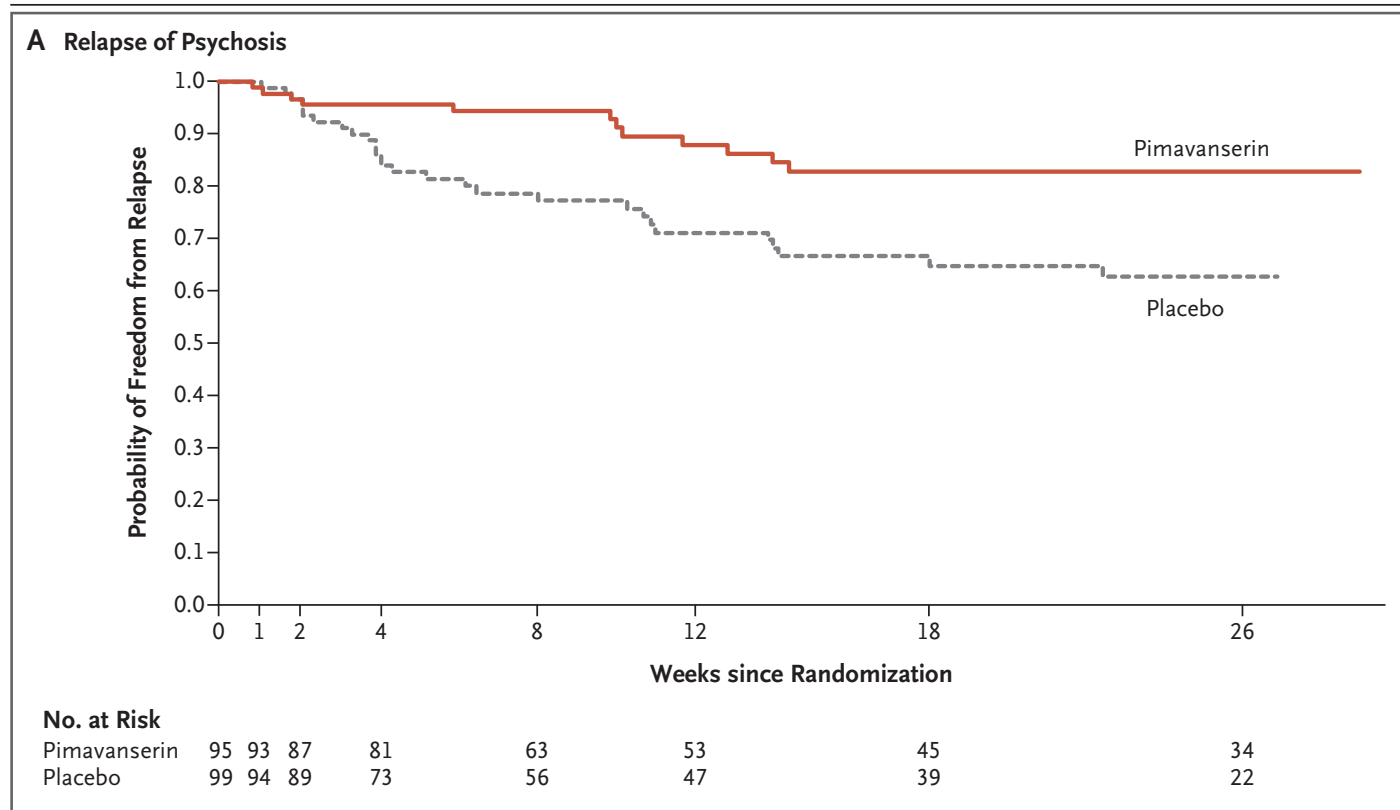

B Trial Discontinuation for Any Reason

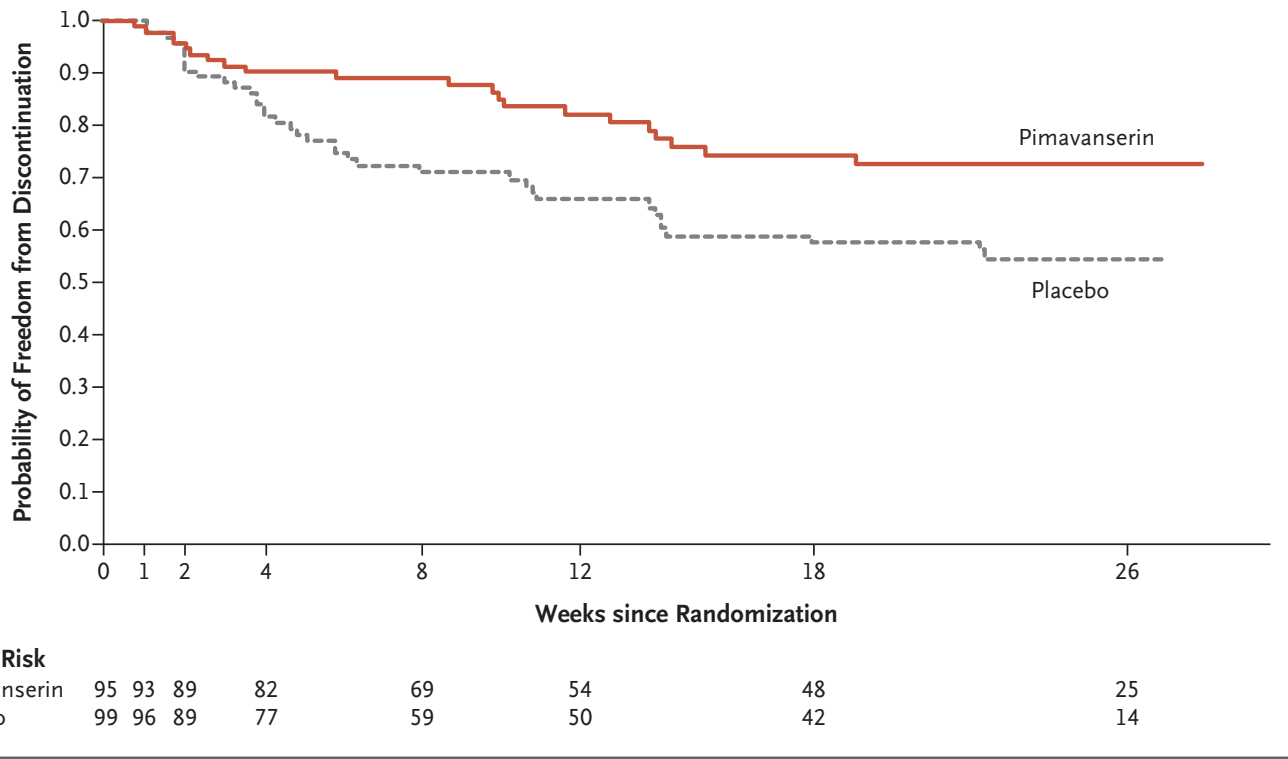

Figure 2. Relapse of Psychosis and Trial Discontinuation.

Shown are the results at the time of the interim analysis. Panel A shows Kaplan-Meier curves for the risk of relapse, assessed in a time-to-event analysis, during the double-blind phase among the 95 patients who had been randomly assigned to continue receiving pimavanserin and the 99 patients who had been assigned to receive placebo. Analysis of the differences in Kaplan-Meier probabilities at weeks 1, 2, 4, 8, 12, 18, and 26 showed statistical significance of the separation at the nominal $5 \%$ level at week 4 and for all time points thereafter. Panel B shows Kaplan-Meier curves for trial discontinuation for any reason, also assessed in a time-to-event analysis, during the double-blind phase.

among those who were switched to placebo, with ity to assess future treatment response in patients an estimated difference of 16 percentage points. who did not meet the full response criteria at The risk of trial discontinuation for any reason week 8. Because the trial was stopped early for was lower with pimavanserin than with placebo.

This trial has limitations. Requiring sustained response in the open-label phase limited the abilefficacy, the ability to assess clinical predictors of relapse is diminished, and it is possible that the active-treatment or placebo group would have 
Table 3. Adverse Events.*

\begin{tabular}{|c|c|c|c|}
\hline \multirow[t]{3}{*}{ Adverse Event } & Open-Label Phase & \multicolumn{2}{|c|}{ Double-Blind Phase } \\
\hline & $\begin{array}{l}\text { Pimavanserin } \\
\quad(\mathrm{N}=392)\end{array}$ & $\begin{array}{l}\text { Pimavanserin } \\
\quad(\mathrm{N}=105)\end{array}$ & $\begin{array}{c}\text { Placebo } \\
(\mathrm{N}=112)\end{array}$ \\
\hline & \multicolumn{3}{|c|}{ number of patients (percent) } \\
\hline Any adverse event & $142(36.2)$ & $43(41.0)$ & $41(36.6)$ \\
\hline Serious adverse event $\uparrow$ & $20(5.1)$ & $5(4.8)$ & $4(3.6)$ \\
\hline Adverse event related to pimavanserin or placebo & $45(11.5)$ & $9(8.6)$ & $10(8.9)$ \\
\hline Adverse event leading to trial discontinuation & $30(7.7)$ & $3(2.9)$ & $4(3.6)$ \\
\hline Adverse event resulting in death $\int$ & $1(0.3)$ & $1(1.0)$ & 0 \\
\hline \multicolumn{4}{|l|}{ Individual adverse events $\emptyset$} \\
\hline Anxiety & $6(1.5)$ & $3(2.9)$ & 0 \\
\hline Asthenia & $3(0.8)$ & $3(2.9)$ & $1(0.9)$ \\
\hline Confusional state & $8(2.0)$ & $1(1.0)$ & 0 \\
\hline Constipation & $10(2.6)$ & $2(1.9)$ & $1(0.9)$ \\
\hline Diarrhea & $5(1.3)$ & 0 & $3(2.7)$ \\
\hline Dizziness & $6(1.5)$ & $3(2.9)$ & 0 \\
\hline Headache & $6(1.5)$ & $10(9.5)$ & $5(4.5)$ \\
\hline Hypertension & $9(2.3)$ & $2(1.9)$ & $2(1.8)$ \\
\hline Nasopharyngitis & $7(1.8)$ & $1(1.0)$ & $4(3.6)$ \\
\hline Nausea & $8(2.0)$ & 0 & $2(1.8)$ \\
\hline Prolonged QT interval\| & $2(0.5)$ & $3(2.9)$ & 0 \\
\hline Urinary tract infection & $20(5.1)$ & $7(6.7)$ & $4(3.6)$ \\
\hline Weight decreased & $5(1.3)$ & $1(1.0)$ & $3(2.7)$ \\
\hline
\end{tabular}

* Shown are treatment-emergent adverse events that occurred in at least $2 \%$ of the patients in the open-label phase or in either trial group in the double-blind phase. A treatment-emergent adverse event is an adverse event with an onset on or after the date of the first trial dose and no later than the date of the last trial dose plus 30 days. For patients who underwent randomization in the double-blind phase, if the onset of the adverse event was on or after the date of first dose in the double-blind phase, the adverse event was assigned to the double-blind phase.

$\dagger$ A serious adverse event was an adverse event that met one or more of the following criteria: was fatal or life-threatening, resulted in disability or permanent damage, led to hospitalization, prolonged existing hospitalization, was a congenital anomaly or birth defect, or was medically significant. The classification of an adverse event as serious was made by the investigator.

$\mp$ An adverse event was considered to be related to pimavanserin or placebo by the investigator if there was a reasonable possibility that the event may have been caused by the trial treatment under investigation. Events with a missing relationship were classified as being related.

$\int A$ 75-year-old White man died during the open-label phase from suspected myocardial infarction, which was considered to be unrelated to the trial drug by the investigator. An 81-year-old White man who was assigned to receive pimavanserin in the double-blind phase died from septic and metabolic encephalopathy caused by a dental abscess, which was considered to be unrelated to drug therapy by the investigator.

9 No significant between-group differences in adverse events were observed when tested at the $5 \%$ level.

\| The adverse events involving prolongation of the corrected QT interval, calculated with the use of Fridericia's formula, involved maximum changes from baseline of $12 \mathrm{msec}$ and $51 \mathrm{msec}$ in two patients in the open-label phase (the former was in a patient who discontinued the trial owing to lack of response, and the latter resolved with dose reduction) and $6 \mathrm{msec}, 22 \mathrm{msec}$, and $77 \mathrm{msec}$ in three patients in the double-blind phase (the first two resolved without intervention, and the third remained stable until the patient discontinued the trial). All events were detected on electrocardiography and were asymptomatic.

had additional relapse events or adverse events if the trial had been longer. Pimavanserin has shown efficacy in patients with hallucinations and delusions associated with Parkinson's disease psychosis and is approved for that indication, and approximately $15 \%$ of the patients in the trial had Parkinson's disease, which may have skewed the results in favor of pimavanserin. Previous trials that involved patients with Parkinson's disease-related psychosis included only those with normal cognition or with MMSE scores of 21 or more. In contrast, $61 \%$ of the patients with Par- 
kinson's disease in the current trial had moderate-to-severe dementia. Almost all the patients in the trial were White. Finally, the double-blind phase was planned for 26 weeks of follow-up of each individual patient but was stopped early for efficacy, which contributed to a median duration of exposure during this phase of approximately 18 weeks in the pimavanserin group and 11 weeks in the placebo group.

Common adverse events that occurred more frequently with pimavanserin than with placebo were headache, constipation, and urinary tract infection. The mean change in QTcF in patients who were exposed to pimavanserin in the openlabel phase was $5.4 \mathrm{msec}$, consistent with the current labeling of the drug, and five adverse events involving asymptomatic prolongation of the QT interval were reported.

In this trial that involved patients with psychosis related to several types of neurodegenerative disease and that was stopped early for efficacy, discontinuation of pimavanserin in patients who had previously had a response to the drug led to a higher risk of relapse of psychosis than did continued receipt of pimavanserin.

Supported by Acadia Pharmaceuticals.

Disclosure forms provided by the authors are available with the full text of this article at NEJM.org.

A data sharing statement provided by the authors is available with the full text of this article at NEJM.org.

We thank Richard S. Perry, Pharm.D., who was paid by Acadia Pharmaceuticals, and Randall Owen, M.D., an employee of Acadia Pharmaceuticals at the time of this trial, for editorial assistance with an earlier version of the manuscript.

\section{REFERENCES}

1. Gale SA, Acar D, Daffner KR. Dementia. Am J Med 2018;131:1161-9.

2. Jellinger KA. Cerebral correlates of psychotic syndromes in neurodegenerative diseases. J Cell Mol Med 2012;16:995-1012.

3. Reus VI, Fochtmann LJ, Eyler AE, et al. The American Psychiatric Association practice guideline on the use of antipsychotics to treat agitation or psychosis in patients with dementia. Am J Psychiatry 2016;173: 543-6.

4. Aarsland D, Larsen JP, Tandberg E, Laake K. Predictors of nursing home placement in Parkinson's disease: a population-based, prospective study. J Am Geriatr Soc 2000;48:938-42.

5. Lyketsos CG, Colenda CC, Beck C, et al. Position statement of the American Association for Geriatric Psychiatry regarding principles of care for patients with dementia resulting from Alzheimer disease. Am J Geriatr Psychiatry 2006;14:561-72.

6. Peters ME, Schwartz S, Han D, et al. Neuropsychiatric symptoms as predictors of progression to severe Alzheimer's dementia and death: the Cache County Dementia Progression Study. Am J Psychiatry 2015;172:460-5.

7. Vernon EK, Cooley B, Rozum W, et al. Caregiver-care recipient relationship closeness is associated with neuropsychiatric symptoms in dementia. Am J Geriatr Psychiatry 2019;27:349-59.

8. Lornstad MT, Aarфen M, Bergh S, Benth JŠ, Helvik A-S. Prevalence and persistent use of psychotropic drugs in older adults receiving domiciliary care at baseline. BMC Geriatr 2019;19:119.

9. Deberdt WG, Dysken MW, Rappaport SA, et al. Comparison of olanzapine and risperidone in the treatment of psychosis and associated behavioral disturbances in patients with dementia. Am J Geriatr Psychiatry 2005;13:722-30.

10. Mintzer J, Greenspan A, Caers I, et al. Risperidone in the treatment of psychosis of Alzheimer disease: results from a pro- spective clinical trial. Am J Geriatr Psychiatry 2006;14:280-91.

11. Mintzer JE, Tune LE, Breder CD, et al. Aripiprazole for the treatment of psychoses in institutionalized patients with $\mathrm{Alz}$ heimer dementia: a multicenter, randomized, double-blind, placebo-controlled assessment of three fixed doses. Am J Geriatr Psychiatry 2007;15:918-31.

12. Tampi RR, Tampi DJ, Balachandran S, Srinivasan S. Antipsychotic use in dementia: a systematic review of benefits and risks from meta-analyses. Ther Adv Chronic Dis 2016;7:229-45.

13. Tariot PN, Schneider L, Katz IR, et al. Quetiapine treatment of psychosis associated with dementia: a double-blind, randomized, placebo-controlled clinical trial. Am J Geriatr Psychiatry 2006;14:767-76.

14. Hacksell U, Burstein ES, McFarland $\mathrm{K}$, Mills RG, Williams H. On the discovery and development of pimavanserin: a novel drug candidate for Parkinson's psychosis. Neurochem Res 2014;39:2008-17. 15. Cummings J, Isaacson S, Mills R, et al. Pimavanserin for patients with Parkinson's disease psychosis: a randomised, placebocontrolled phase 3 trial. Lancet 2014;383: 533-40.

16. Espay AJ, Guskey MT, Norton JC, et al. Pimavanserin for Parkinson's disease psychosis: effects stratified by baseline cognition and use of cognitive-enhancing medications. Mov Disord 2018;33:1769-76.

17. Ballard C, Banister C, Khan Z, et al. Evaluation of the safety, tolerability, and efficacy of pimavanserin versus placebo in patients with Alzheimer's disease psychosis: a phase 2, randomised, placebocontrolled, double-blind study. Lancet Neurol 2018;17:213-22.

18. Devanand DP, Mintzer J, Schultz SK, et al. Relapse risk after discontinuation of risperidone in Alzheimer's disease. N Engl J Med 2012;367:1497-507.

19. Ballard C, Brown R, Fossey J, et al. Brief psychosocial therapy for the treat- ment of agitation in Alzheimer disease (the CALM-AD trial). Am J Geriatr Psychiatry 2009;17:726-33.

20. McKhann GM, Knopman DS, Chertkow $\mathrm{H}$, et al. The diagnosis of dementia due to Alzheimer's disease: recommendations from the National Institute on AgingAlzheimer's Association workgroups on diagnostic guidelines for Alzheimer's disease. Alzheimers Dement 2011;7:263-9.

21. Emre M, Aarsland D, Brown R, et al. Clinical diagnostic criteria for dementia associated with Parkinson's disease. Mov Disord 2007;22:1689-707.

22. McKeith IG, Dickson DW, Lowe J, et al. Diagnosis and management of dementia with Lewy bodies: third report of the DLB Consortium. Neurology 2005;65:1863-72. 23. Rascovsky K, Hodges JR, Knopman D, et al. Sensitivity of revised diagnostic criteria for the behavioural variant of frontotemporal dementia. Brain 2011;134:2456-77. 24. Sachdev P, Kalaria R, O'Brien J, et al. Diagnostic criteria for vascular cognitive disorders: a VASCOG statement. Alzheimer Dis Assoc Disord 2014;28:206-18.

25. Folstein MF, Folstein SE, McHugh PR. "Mini-mental state": a practical method for grading the cognitive state of patients for the clinician. J Psychiatr Res 1975;12: 189-98.

26. Andreasen N. Scale for the Assessment of Positive Symptoms (SAPS). Iowa City: University of Iowa, 1984.

27. Guy W. Clinical global impressions. In: ECDEU assessment manual for psychopharmacology. Rev. ed. Rockville, MD: Department of Health, Education, and Welfare, 1976:218-22. (DHEW publication no. ADM 76-338.)

28. Chouinard G, Margolese HC. Manual for the Extrapyramidal Symptom Rating Scale (ESRS). Schizophr Res 2005;76:247-65. 29. Lin DY, Wei LJ. The robust inference for the proportional hazards model. J Am Stat Assoc 1989;84:1074-8.

Copyright (c) 2021 Massachusetts Medical Society. 